\title{
Brainstem Infarction and Panuveitis due to Sarcoidosis Successfully Treated with Steroid Pulse Therapy
}

\author{
Natsuyo Yoshida-Hata, ${ }^{1}$ Shigeko Yashiro, ${ }^{1}$ Noritoshi Arai, ${ }^{2}$ and Sousuke Takeuchi ${ }^{2}$ \\ ${ }^{1}$ Department of Ophthalmology, National Center for Global Health and Medicine, Tokyo 162-8655, Japan \\ ${ }^{2}$ Department of Neurology, National Center for Global Health and Medicine, Tokyo 162-8655, Japan \\ Correspondence should be addressed to Natsuyo Yoshida-Hata, knmiyu0717@yahoo.co.jp
}

Received 23 August 2011; Accepted 4 November 2011

Academic Editor: Huy M. Do

Copyright ( 2012 Natsuyo Yoshida-Hata et al. This is an open access article distributed under the Creative Commons Attribution License, which permits unrestricted use, distribution, and reproduction in any medium, provided the original work is properly cited.

\begin{abstract}
A 36-year-old man visited our hospital because of blurred vision and redness of the conjunctiva. Slit-lamp examination showed panuveitis. Two days later, he suddenly experienced dizziness, speech disturbance, paralysis of his right extremities, and gait disturbances. Neurological examinations suggested that his symptoms were caused by a left lateral medullary lesion. He also had erythema mainly on his trunk. Magnetic resonance imaging (MRI) of his brain demonstrated a small infarct on the left side of the medulla oblongata. Clinical presentation and MRI findings were consistent with the diagnosis of a Wallenberg's syndrome. He also had bilateral hilar lymphadenopathy. A skin biopsy showed granulomatous nodular dermatitis compatible with sarcoidosis. He was treated with steroid pulse therapy and his neurological and ocular symptoms immediately improved. Only seven similar cases of intracranical sarcoidosis have been reported, but none had been treated with steroid pulse therapy. We recommend that steroid pulse therapy be considered to treat patients with sarcoidosis with signs of lesions in the central nervous system.
\end{abstract}

\section{Introduction}

Sarcoidosis is characterized by noncaseating granulomas, and in $5 \%$ of the patients, the lesions are located in the central nervous system [1]. However, strokes are uncommon in patients with sarcoidosis in spite of small vessel inflammation, and sarcoidosis in the medulla oblongata is rare [24]. Sarcoidosis is a systemic disease associated with uveitis and accounts for $10 \%$ of the uveitis cases in Japan $[5,6]$. Ocular sarcoidosis is managed well by systemic corticosteroid therapy and is generally easier to manage than eyes with Bechet disease [7].

Chronic posterior ocular sarcoidosis is more difficult to manage, and it can lead to sight-threatening complications, for example, cataracts, glaucoma, and cystoid macular oedema. Eyes with posterior uveitis with chronic sarcoidosis have a poor visual prognosis despite immunosuppressive therapy [8].

Corticosteroids, usually combined with immnosuppresive agents, remain the mainstay therapy for ocular sarcoidosis although refractory cases do occur [9]. The refractory cases generally have a poor visual prognosis.
We described a patient with ocular sarcoidosis and a cerebral infarction, who had an excellent response to steroidpulse therapy.

\section{Case Report}

A 36-year-old man reported blurred vision and redness of the conjunctiva in both eyes. He was referred to our hospital in January 2009, and our examination showed that his bestcorrected visual acuity (BCVA) was $1.2(20 / 20)$ in both eyes. Slit-lamp examination showed ciliary injection and keratic precipitates. Fundus examination demonstrated vasculitis in both eyes (Figures 1(a) and 1(b)).

Physical examination showed exudative erythema nodosum on his trunk and both thighs. Chest X-rays and CT scans demonstrated bilateral hilar and mediastinal lymph node swelling. Laboratory tests, including antibodies for rheumatoid factor, Sjögren syndrome, double-stranded DNA, and antinuclear antibodies, were negative. In addition, the serum antiotensin-converting enzyme level was high at $27.0 \mathrm{U} / \mathrm{L}$ (normal $<20$ ). 


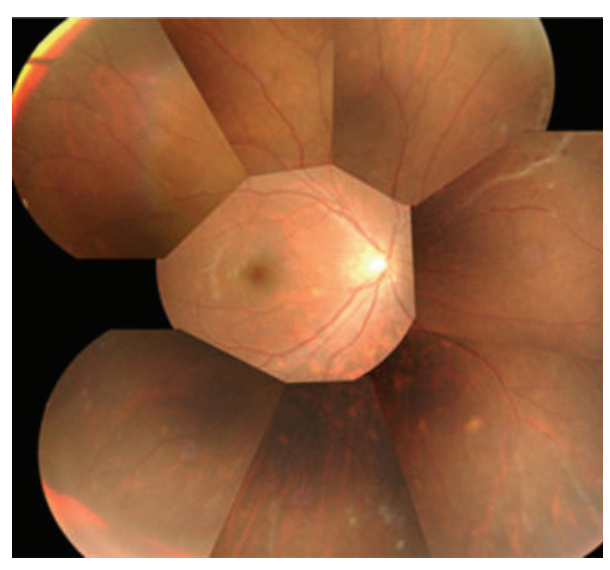

(a)

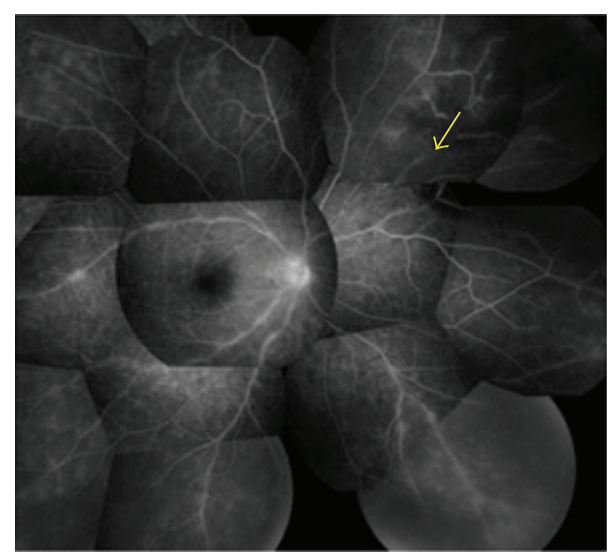

(c)

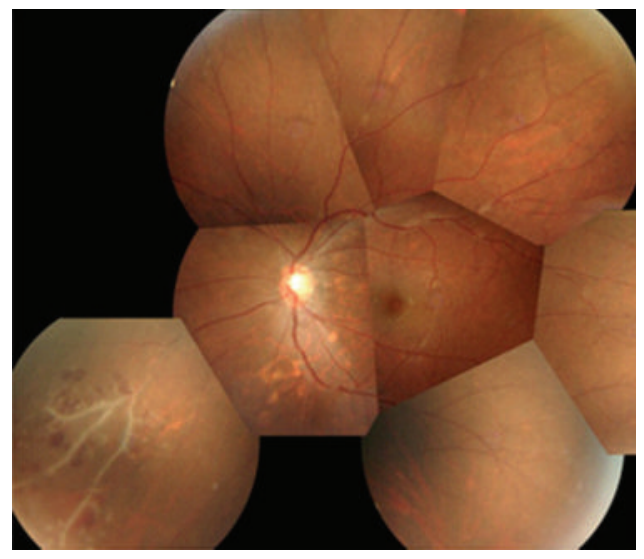

(b)

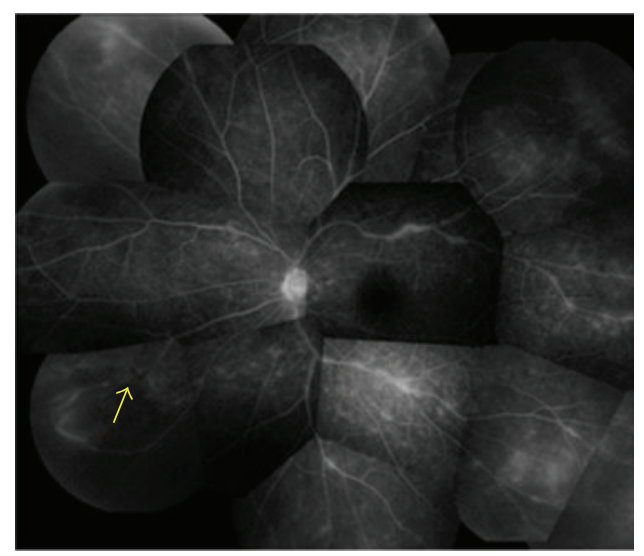

(d)

FIGURE 1: Composite photographs of the fundus and fluorescein angiograms of a patient with ocular sarcoidosis with an infarct in the medulla oblongata. (a) and (b) Infiltrates and ischemic areas can be seen. (c) and (d) Fluorescein angiograms of right and left eyes showing areas of nonperfusion (arrow) in both eyes.

Two days after his first visit, he reported dizziness, speech disturbance, clumsiness of his right extremities, and gait disturbances. Neurological examinations showed left ptosis and miosis, hoarseness, dysphagia, right mild hemiparesis, left ataxia of his extremities, and truncal ataxia. He also had hypalgesia/hypotemperature on his right side. These findings suggested a left Wallenberg syndrome. Brain magnetic resonance imaging (MRI) showed an infarct on the left side of the medulla oblongata (Figure 2). Leptomeningeal enhancement was not observed on MRI. Studies of the cerebrospinal fluid showed normal glycorrhachia $(74 \mathrm{mg} / \mathrm{dL})$, mild lymphocyte elevation, and elevated protein at $69 \mathrm{mg} / \mathrm{dL}$. These findings suggested inflammatory processes in the central nervous systems. Dermal examination/skin biopsy revealed granulomatous nodular dermatitis (Figure 3). 18F-FDG in the acute stage showed an upregulation of both hillar lymphadenitis in the mediastinum and ocular regions.

The patient was diagnosed with neurosarcoidosis with a brainstem infarct. Steroid pulse therapy was prescribed initially with $1000 \mathrm{mg}$ infusion for three days and then slowly tapered. During the pulse therapy, the patient had laser photocoagulation of the nonperfused areas of the retina. The patient had an improvement of his symptoms after the steroid-pulse therapy.
At the last examination on February 2011, the patient was stable with no complications and no recurrence of the ocular and CNS sarcoidosis. And his BCVA was 20/20.

\section{Discussion}

Our findings showed that our patient had CNS sarcoidosis of the medulla oblongata, and steroid-pulse therapy was very effective in resolving the symptoms. The mechanism causing the brain infarction in neurosarcoidosis is not completely known but is thought to result from vasculitis of the small vessels, emboli, or inflammation of the large vessels $[16,17]$. Granulomatous invasion into the blood vessel walls with disruption of the media and internal elastic laminae can cause infarctions of small vessels [18].

Although rare, neurosarcoidosis should be considered in cases of an acute stroke with restricted diffusion of unknown etiology in a young person, even if other stigmata of sarcoidosis are not present. Despite the common finding of vasculitis and microscopic infarction at autopsy, a stroke is usually due to vascular accidents in the cerebral hemisphere and is rarely the presenting sign of neurosarcoidosis (Table 1) $[10,11,15]$. The rarity of brainstem stroke is surprising given 


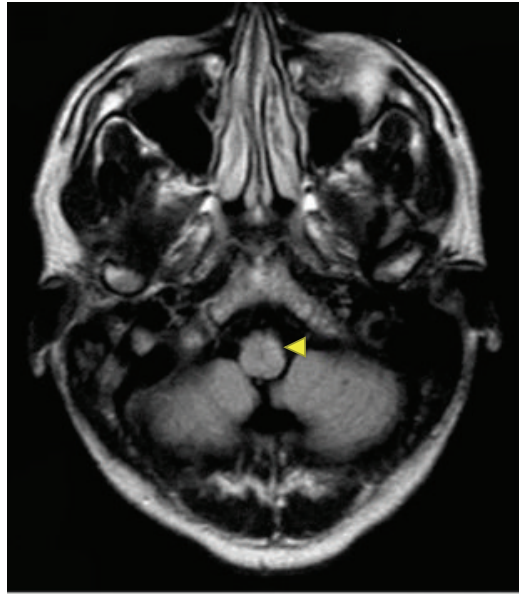

(a)

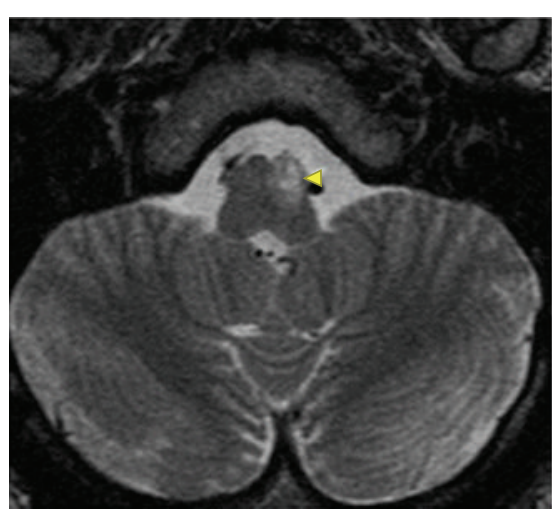

(b)

FIgURE 2: Images of the brain of a patient with an infarct in the medulla oblongata. (a) Left medulla oblongata encephalomalacia on axial T2-fluid-attenuated inversion image (arrow head). (b) Diffusion-weighted (TTt) image showing restricted diffusion in the left medulla oblongata (arrowhead).

TABLE 1: Past reports which reported CNS sarcoidosis as brain ischemic stroke.

\begin{tabular}{llll}
\hline & & Location & Eye symptom \\
\hline Navi, 2009 [10] & $35 \mathrm{M}$ & Pons & None \\
& $46 \mathrm{~F}$ & Pons & None \\
Hodge, 2007 [11] & $36 \mathrm{f}$ & Left frontal subcortical white matter & None \\
Brisman, 2006 [12] & $41 \mathrm{~m}$ & Frontal and lobe & Left eye blindness \\
Nakagaki, 2004 [13] & $75 \mathrm{~m}$ & Right parieto occipital lobes & Ocular sarcoidosis \\
Das, 1998 [14] & $27 \mathrm{f}$ & Infarction of left middle cerebral artery territory & None \\
Michotte, 1991 [15] & $29 \mathrm{~m}$ & Multiple bilateral subcortical lesion & None \\
\hline
\end{tabular}

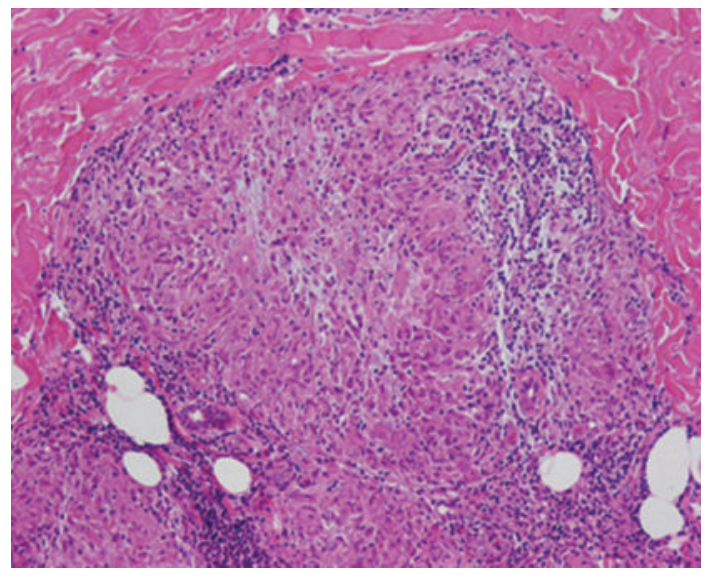

FIGURE 3: Histopathological section of skin biopsy. Nonnecrotizing granuloma with several multinucleated giant cells and epitheliod histiocytes can be seen. Hematoxylin-eosin $\times 250$.

the propensity of basilar inflammation $[10,19]$. To the best of our knowledge, our report is the first case of neurosarcoidosis with a brainstem infarction in the medulla oblongata.

Ocular sarcoidosis is not rare in Japan, and it has been found that sarcoidosis responds well to steroid therapy. But
$10 \%$ of ocular sarcoidosis required periocular corticosteroids to treat the uveitis, and $13 \%$ of ocular sarcoidosis required systemic treatment for both the ocular and systemic disease [19]. In addition, $8 \%$ of patients required immunosuppressive therapy because steroids alone did not control the inflammation or the dose required to prevent a relapse of uveitis was too high [19]. Poor visual outcome ranged from $9 \%$ to $66 \%[19,20]$.

The management of resistant uveitis associated with sarcoidosis is a serious therapeutic problem. The toxicity from steroids such as osteoporosis, femur head necrosis, infection, steroidgenic diabetes, corticosteroid-induced glaucoma, and steroid-induced psychosis is difficult to control. This is why clinicians resist the use of high-dose steroids for long periods. Thus, it is important to know that steroid-pulse therapy is effective in treating the ocular inflammation due to sarcoidosis. Because steroid-pulse therapy has fewer sideeffect, we recommend steroid pulse therapy for ocular and CNS sarcoidosis.

\section{Acknowledgment}

The authors thank Professor Duco Hamasaki for editing this manuscript. 


\section{References}

[1] J. P. Zajicek, N. J. Scolding, O. Foster et al., "Central nervous system sarcoidosis-diagnosis and management," Quarterly Journal of Medicine, vol. 92, no. 2, pp. 103-117, 1999.

[2] T. G. B. Mahadewa, H. Nakagawa, T. Watabe, and T. Inoue, "Intramedullary neurosarcoidosis in the medulla oblongata: a case report," Surgical Neurology, vol. 61, no. 3, pp. 283-287, 2004.

[3] E. Hayashi, A. Niwa, Y. Narita, and S. Kuzuhara, "[A case of sarcoidosis with simultaneous involvement of the lower brainstem and the whole cervical cord and the extraocular muscles]," Rinsho Shinkeigaku, vol. 41, pp. 314-317, 2001.

[4] M. Graf, A. Wakhloo, K. Schmidtke, H. Bloss, and B. Volk, "Sarcoidosis of the spinal cord and medulla oblongata. A pathological and neuroradiological case report," Clinical Neuropathology, vol. 13, no. 1, pp. 19-25, 1994.

[5] Y. Asukata, M. Ishihara, Y. Hasumi et al., "Guidelines for the diagnosis of ocular sarcoidosis," Ocular Immunology and Inflammation, vol. 16, no. 3, pp. 77-81, 2008.

[6] H. Goto, M. Mochizuki, K. Yamaki, S. Kotake, M. Usui, and S. Ohno, "Epidemiological survey of intraocular inflammation in Japan," Japanese Journal of Ophthalmology, vol. 51, no. 1, pp. 41-44, 2007.

[7] R. B. Nussenblat and S. M. Whitcup, Uveitis: Fundamentals and Clinical Practice: Expert Consult Online and Print, Mosby, 2010.

[8] D. A. Jabs and C. J. Johns, "Ocular involvement in chronic sarcoidosis," American Journal of Ophthalmology, vol. 102, no. 3, pp. 297-301, 1986.

[9] N. P. Jones, "Sarcoidosis and uveitis," Ophthalmology Clinics of North America, vol. 15, no. 3, pp. 319-326, 2002.

[10] B. B. Navi and L. M. Deangelis, "Sarcoidosis presentiing as brainstem ischemic stroke," Neurology, vol. 72, no. 11, pp. 1021-1022, 2009.

[11] M. H. Hodge, R. L. Williams, and M. B. Fukui, "Neurosarcoidosis presenting as acute infarction on diffusion-weighted MR imaging: summary of radiologic findings," American Journal of Neuroradiology, vol. 28, no. 1, pp. 84-86, 2007.

[12] J. L. Brisman, A. Hinduja, J. S. McKinney, and B. Gerhardstein, "Successful emergent angioplasty of neurosarcoid vasculitis presenting with strokes," Surgical Neurology, vol. 66, no. 4, pp. 402-404, 2006.

[13] H. Nakagaki, J. -I. Furuya, T. Nagata et al., "An elder case of neurosarcoidosis associated with brain infarction," Clinical Neurology, vol. 44, no. 2, pp. 81-85, 2004.

[14] S. K. Das, I. Sinha, T. N. Kundu, K. Sanyal, V. Santosh, and S. K. Shankar, "Two cases of neurosarcoidosis presenting as peripheral neuropathy and stroke in young," Journal of Association of Physicians of India, vol. 46, no. 5, pp. 479-481, 1998.

[15] A. Michotte, P. Dequenne, D. Jacobovitz, and J. Hildebrand, "Focal neurological deficit with sudden onset as the first manifestation of sarcoidosis: a case report with MRI followup," European Neurology, vol. 31, no. 6, pp. 376-379, 1991.

[16] A. M. Corse and B. J. Stern, "Neurosarcoidosis and stroke," Stroke, vol. 21, no. 1, pp. 152-153, 1990.

[17] D. S. Younger, A. P. Hays, J. C. M. Brust, and L. P. Rowland, "Granulomatous angiitis of the brain. An inflammatory reaction of diverse etiology," Archives of Neurology, vol. 45, no. 5, pp. 514-518, 1988.

[18] M. M. Brown, A. J. Thompson, J. A. Wedzicha, and M. Swash, "Sarcoidosis presenting with stroke," Stroke, vol. 20, no. 3, pp. 400-405, 1989.
[19] A. Lobo, K. Barton, D. Minassian, R. M. Du Bois, and S. Lightman, "Visual loss in sarcoid-related uveitis," Clinical and Experimental Ophthalmology, vol. 31, no. 4, pp. 310-316, 2003.

[20] B. A. Cruz, D. D. Reis, and C. A. Araujo, "Refractory retinal vasculitis due to sarcoidosis successfully treated with infliximab," Rheumatology International, vol. 27, no. 12, pp. 1181-1183, 2007. 


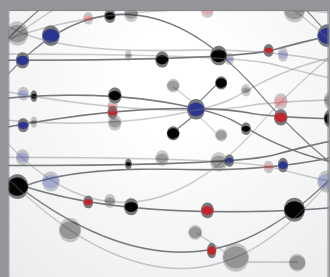

The Scientific World Journal
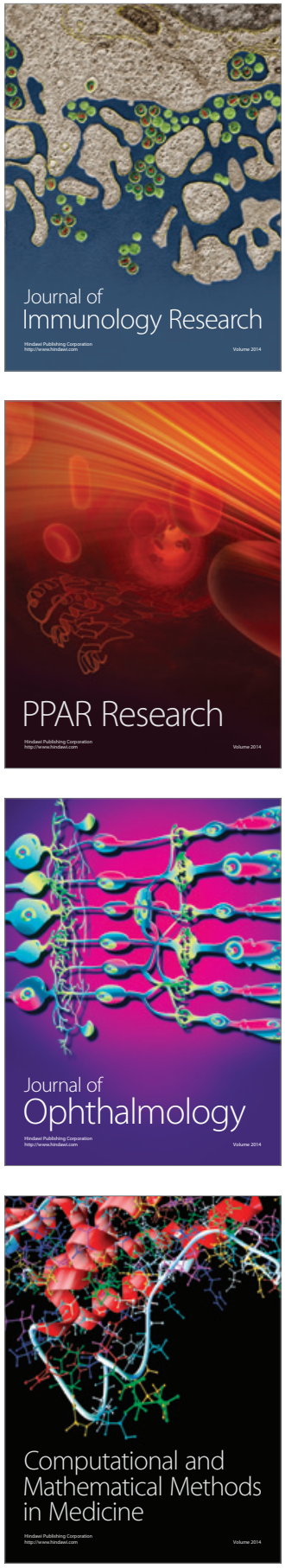

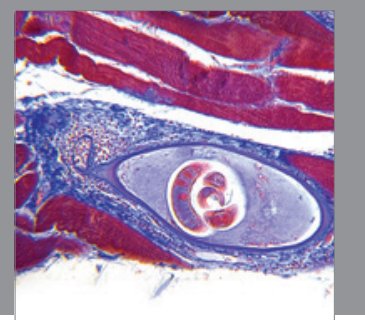

Gastroenterology

Research and Practice
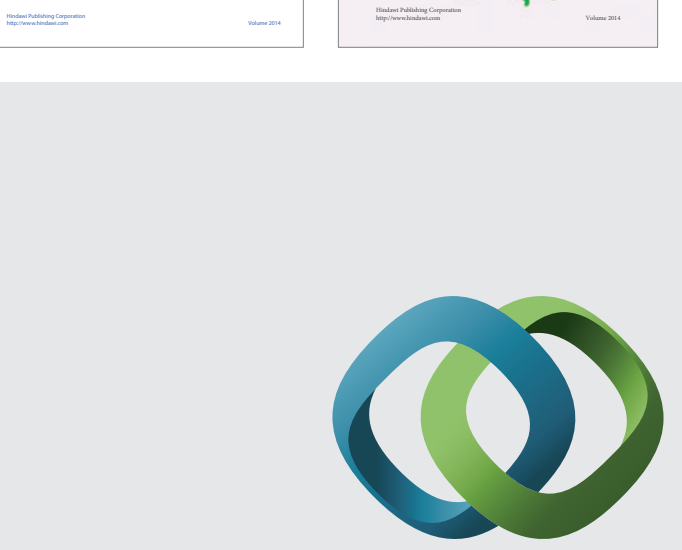

\section{Hindawi}

Submit your manuscripts at

http://www.hindawi.com
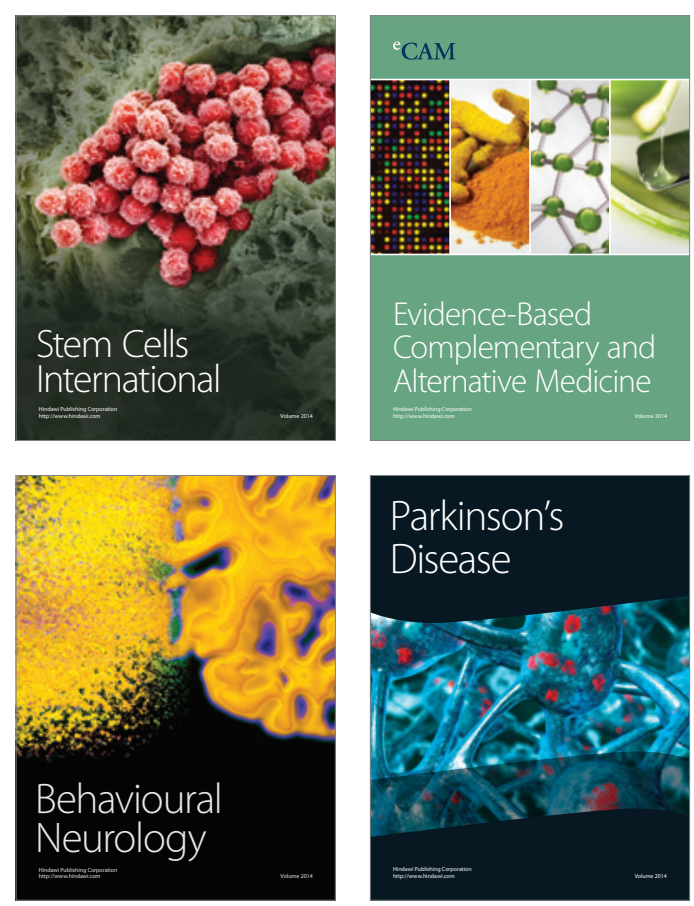

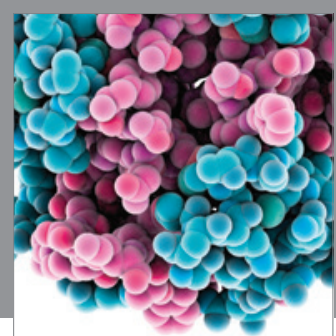

Journal of
Diabetes Research

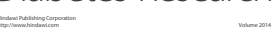

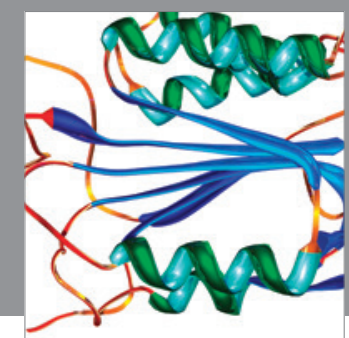

Disease Markers
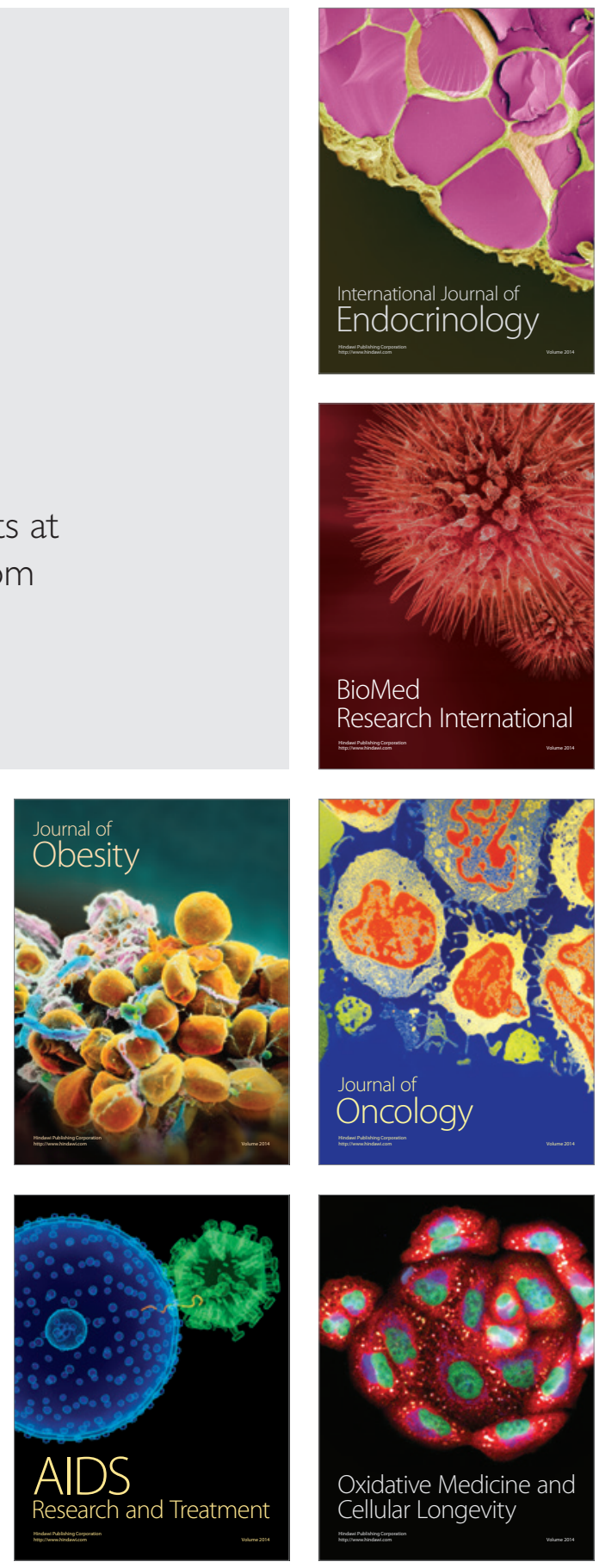